\title{
Impact of Pregnancy on Intra-Host Genetic Diversity of Influenza A Viruses in Hospitalised Women: A Retrospective Cohort Study
}

\author{
Gregory Destras 1,2,3, Maxime Pichon 1,2,3@ Bruno Simon 2 ${ }^{-1}$, Martine Valette ${ }^{2,3}$, \\ Vanessa Escuret 1,2,3, Pierre-Adrien Bolze 4, Gil Dubernard ${ }^{5}$, Pascal Gaucherand ${ }^{6}$, \\ Bruno Lina $1,2,3$ (D) and Laurence Josset $1,2,3, *$ (D) \\ 1 Virpath, INSERM U1111, CNRS UMR5308, International Center for Infectiology Research, ENS Lyon, \\ Claude Bernard Lyon 1 University, 69008 Lyon, France; gregory.destras@chu-lyon.fr (G.D.); \\ maxime.pichon@chu-poitiers.fr (M.P.); vanessa.escuret@chu-lyon.fr (V.E.); bruno.lina@chu-lyon.fr (B.L.) \\ 2 Virology Laboratory, Infectious Agents Institute, CBN, Groupement Hospitalier Nord, Hospices Civils de \\ Lyon, 69004 Lyon, France; sib0.smb@gmail.com (B.S.); martine.valette@chu-lyon.fr (M.V.) \\ 3 Centre National des Virus des infections Respiratoires, Infectious Agents Institute, CBN, Groupement \\ Hospitalier Nord, 69004 Lyon, France \\ 4 Service de Chirurgie Gynécologique et Oncologique-Obstétrique, Centre Hospitalier Lyon Sud, \\ Hospices Civils de Lyon, 69310 Pierre-Bénite, France; pierre-adrien.bolze@chu-lyon.fr \\ 5 Hospices Civils de Lyon, Service de Gynécologie et d'Obstétrique, Hôpital de la Croix Rousse, \\ 69004 Lyon, France; gil.dubernard@chu-lyon.fr \\ 6 Consultation Obstétrique, Groupement Hospitalier Est, Hospices Civils de Lyon, 69500 Bron, France; \\ pascal.gaucherand@chu-lyon.fr \\ * Correspondence: laurence.josset@chu-lyon.fr; Tel.: +33-(0)4-72-07-10-22
}

Received: 30 September 2019; Accepted: 12 November 2019; Published: 14 November 2019

\begin{abstract}
Characterising dynamics of Influenza A Viruses (IAV) within-host evolution is an active field of research which may lead to a better understanding of viral pathogenesis. Using a pregnant mouse model, a study has recently suggested that immune modulation during pregnancy could promote the emergence of IAV quasispecies with increased virulence. Herein, we assess the clinical relevance of these findings in humans. We studied IAV intra-host diversity (ihD) in pregnant $(n=36)$ and non-pregnant $(n=23)$ women hospitalized in Lyon for IAV infection $(01 / 2015-05 / 2018)$. Whole IAV genomes present in nasopharyngeal samples were sequenced in duplicate to analyze reproducible intra-host single nucleotide variants (ihSNV). Counts, relative frequencies and locations of ihSNV were used as indicators of $\mathrm{ihD}$. The median ihSNV/kb counts per segment were between 0 and 1.3 . There was $>81 \%$ ihSNV at relative frequencies between $1-5 \%$ for $\mathrm{H} 1 \mathrm{~N} 1$ and $>51 \%$ for H3N2 IAV. No significant difference was noted between pregnant and non-pregnant women when considering all or only non-synonymous ihSNV. Seven convergent non-synonymous ihSNV were found; none were significantly associated with pregnancy. These results suggest that modulation of the immune system during pregnancy in humans does not impact IAV ihD, in contrast to mice.
\end{abstract}

Keywords: influenza; pregnancy; quasispecies; ultra-deep sequencing; viral diversity

\section{Introduction}

Due to its error-prone polymerase, RNA eight-segmented influenza A viruses (IAV) acquire genetic diversity when replicating [1]. Understanding intra-host diversity (ihD) of IAV is of great interest because ihD may shape IAV evolution at the inter-host scale but also impact IAV pathogenesis [2]. Several factors, including host immune competence, might modulate ihD. Recently, using allogeneic 
pregnant mice infected with human $\mathrm{A}(\mathrm{H} 1 \mathrm{~N} 1) \mathrm{pdm} 09$, Engels et al. suggested that immune modifications of both innate and adaptive immunity during pregnancy could drive the emergence of convergent non-synonymous mutations (Q223R on HA according to HA1 numbering, R211K on NS1, and R54N on NEP) associated with fatal outcomes [3]. They postulated that this mechanism could explain why pregnant women are more at risk of developing severe influenza. To examine the clinical relevance of these findings, we assessed IAV ihD in women according to pregnancy status and its potential impact on fostering severe forms.

\section{Materials and Methods}

\subsection{Study Cohort}

All women aged 15-45 years admitted to the emergency departments of the Lyon university hospital (France) with nasopharyngeal swabs positive for IAV during three consecutive influenza seasons (01/2015-05/2018) were retrospectively identified. Women with missing clinical record and/or samples were excluded. Patients were classified according to pregnancy status and influenza severity (defined as the need for mechanical ventilation and/or cardiac complication-myocarditis or pericarditis).

\subsection{IAV Whole Genome Amplification and Sequencing}

IAV whole genome amplification was performed directly on IAV-positive samples as previously described [4]. Briefly, an automatic extraction platform (Nuclisens EasyMag, Durham, NC, USA) was used, followed by a DNase treatment (TurboDNAse, LifeTechnologies, Carlsbad, California, CA, USA) before whole genome amplification by universal primers (Uni12 and Uni13). Sequencing was performed on a NextSeq $5002 \times 150$ bp reads (Illumina, San Diego, California, US). Importantly, all respiratory samples were extracted and sequenced in duplicate to improve variant detection accuracy. This, however, required a high volume of samples $(400 \mu \mathrm{L})$.

\subsection{Bioinformatic Analysis}

Reads were analyzed as previously described including i) quality trimming and filtering $(>Q 30)$ and removal of human reads; ii) mapping on genome references (A/pH1N1/California/07/2009 and $\mathrm{A} / \mathrm{H} 3 \mathrm{~N} 2 /$ Perth/16/2009) to generate consensus sequences; and iii) intra-host Single Nucleotide Variants (ihSNV) calling using naive variant caller [5]. Genetic diversity was then analyzed using R [6] using the following criteria for each nucleotide position: depth $>1000 \mathrm{X}$; ihSNV detected in both duplicates with mean relative frequency $>1 \%$. Count and relative frequency of ihSNV were normalized by the number of analyzed positions (>1000X).

\subsection{Statistical Analysis}

Statistical analyses were performed using R 3.5.3 (available at https://cran.r-project.org/bin/ windows/base/old/3.5.3/). For qualitative variables, $\chi^{2}$ or G-test was used. For quantitative variables, the $\mathrm{t}$-test or Mann-Whitney test was used as appropriate. Z-scores for ihSNV counts $/ \mathrm{kb}$ were computed per segment for severe cases. A $p$-value $<0.05$ was considered significant.

\subsection{Ethical Statement}

The study was approved by the local ethics committee of the Lyon University Hospital on 21/12/2015. 


\section{Results}

In total, 284 women were identified; after exclusion of those with missing clinical records $(n=12)$ or insufficient volumes of samples $(n=187)$, and after exclusion of sequences with low coverage $(n=26)$, whole genome sequences of 59 IAV (36 pregnant and 23 non-pregnant women) were successfully obtained in duplicate. Pregnant women were significantly younger; there was no significant difference in the frequency of patients with an underlying condition, time since symptom onset, antiviral treatment before sampling, hospitalization for influenza, influenza severity, viral load or viral subtypes between pregnant and non-pregnant women (Table 1).

Table 1. Characteristics of women included in the study.

\begin{tabular}{|c|c|c|c|}
\hline & $\begin{array}{c}\text { Pregnant Women } \\
n=36\end{array}$ & $\begin{array}{l}\text { Non-Pregnant Women } \\
\qquad n=23\end{array}$ & $p$ \\
\hline Age, mean years (SD) & $30.6(0.96)$ & $34.7(1.5)$ & 0.03 \\
\hline \multicolumn{4}{|l|}{ Trimesters, $n(\%)$} \\
\hline Trimester 1 & $2(5.6)$ & & \\
\hline Trimester 2 & $9(25.0)$ & & \\
\hline Trimester 3 & $25(69.4$ & & \\
\hline With underlying condition, $n(\%)$ & $10(30.3)$ & $10(43.5)$ & 0.21 \\
\hline Time since onset of symptoms, median days (IQR) & $2.0(1.0-3.0)$ & $2.0(1.0-3.0)$ & 0.80 \\
\hline Oseltamivir treatment before sampling, $n(\%)$ & $1(2.8)$ & $0(0.0)$ & 1.00 \\
\hline Hospitalisation for influenza, $n(\%)$ & $11(30.6)$ & $11(47.8)$ & 0.18 \\
\hline Severe influenza, $n(\%)$ & $2(5.6)$ & $5(21.7)$ & 0.10 \\
\hline IAV viral load, mean Ct (SD) & $26.01(4.2)$ & $26.35(4.2)$ & 0.80 \\
\hline \multicolumn{4}{|l|}{ IAV sub-type and clades, $n(\%)$} \\
\hline $\mathrm{H} 1 \mathrm{~N} 16 \mathrm{~B} 1$ & $18(50)$ & $13(56.5)$ & 0.96 \\
\hline H3N2 & $18(50)$ & $10(43.5)$ & 0.82 \\
\hline H3N2 3c2a1 & $13(36.1)$ & $9(39.1)$ & 0.65 \\
\hline H3N2 3c2a2 & $3(8.3)$ & $0(0.0)$ & 0.40 \\
\hline H3N2 3c2a3 & $2(5.5)$ & $1(4.3)$ & 0.83 \\
\hline $\mathrm{H} 3 \mathrm{~N} 2 \mathrm{3c} 3 \mathrm{~b}$ & $1(2.8)$ & $0(0.0)$ & 1.00 \\
\hline
\end{tabular}

SD: Standard deviation; IQR: interquartile range; $p$ : $p$-value.

Regarding sequencing results, no significant difference of depth of coverage was found between pregnant and non-pregnant women (Table S1, Figure S1). There was no association between phylogeny and pregnancy or clinical severity (Figures S2-S9).

For diversity, the median ihSNV/kb counts per segment ranged from 0 (for four segments of H1N1 viruses and six segments of H3N2 viruses, in both pregnant and non-pregnant women) to 1.3 for the 5th segment (NP) of H3N2 viruses (Figure 1A,B). There was no significant difference in the number of ihSNV/kb according to pregnancy (Figure 1A,B); this was also found when only non-synonymous ihSNV/kb were considered (Figure S10A,B). Regarding severe cases, all ihSNV z-scores computed for the different segments were lower than 2.6, both for pregnant women (range: -0.95 to 2.56 for H1N1 and -0.57 to 2.53 for H3N2) and non-pregnant women (range: -1.4 to 1.4 for H1N1 and -1.3 to 2.5 for H3N2).

For $\mathrm{H} 1 \mathrm{~N} 1$ viruses, $85 \%$ of the ihSNV were found at a low relative frequency $(1-5 \%)$ in pregnant women and $81 \%$ in non-pregnant women (Figure 1C,D). H3N2 viruses were slightly more diverse; $65 \%$ of the ihSNV were found at a low relative frequency in pregnant women and $51 \%$ in non-pregnant women (Figure 1C,D). Distribution of ihSNV relative frequencies were not significantly different between pregnant and non-pregnant women ( $p=0.16$ for H1N1; $p=0.35$ for H3N2). High-relative frequency ihSNV (>30\%) were all synonymous, except for a G11S mutation in NA that was present in one pregnant woman with severe H1N1 influenza (Figure 1C,D, Figure S10C,D). 

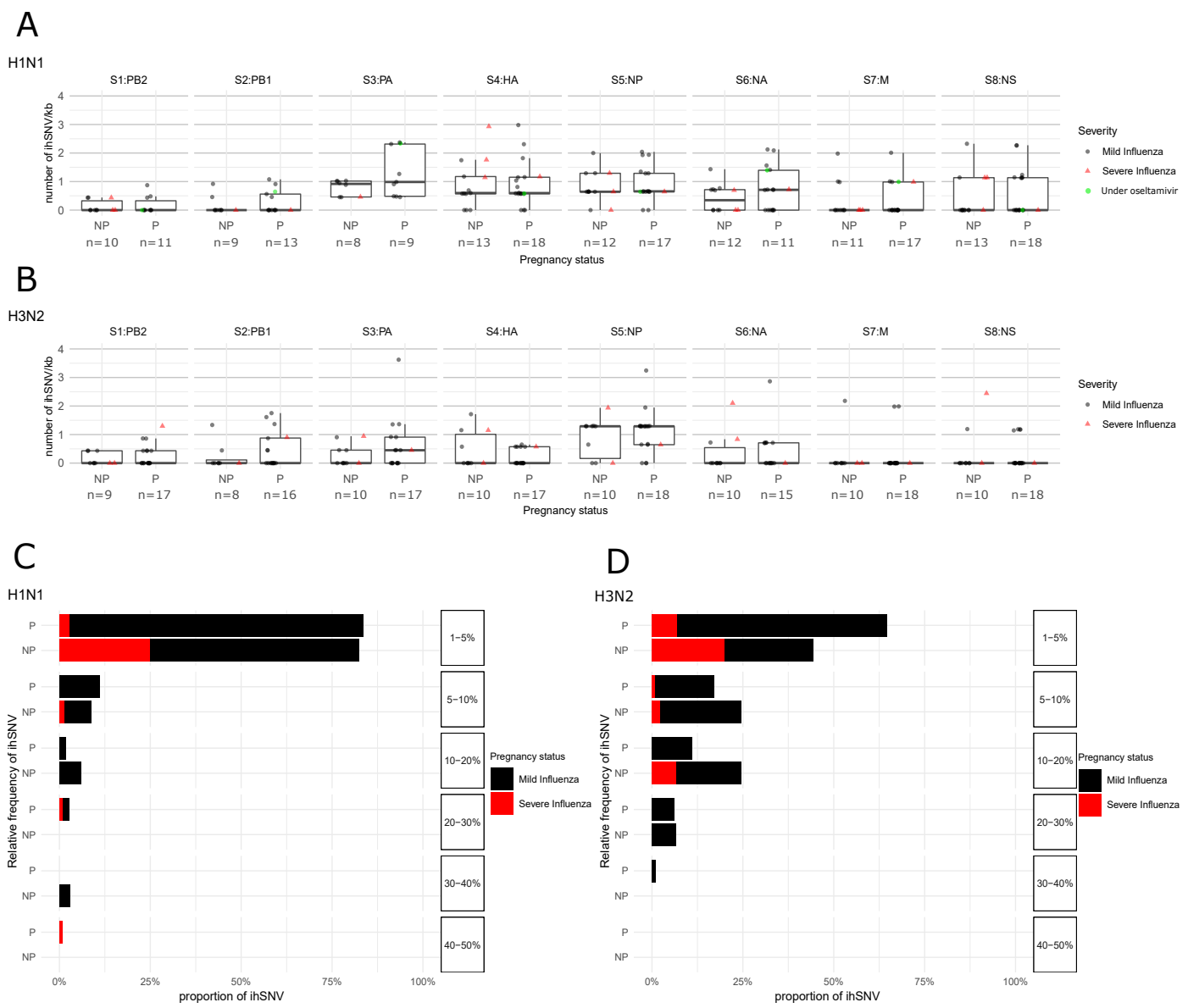

Figure 1. Comparison of Influenza A Viruses intra-host diversity (IAV ihD) between pregnant and non-pregnant women. Number of ihSNV/kb per segment (S1 to S8) in each sample, according to pregnancy status (P: pregnant; NP: non-pregnant) for H1N1 (A) and H3N2 (B) viruses. Each point represents a patient, with black dot for mild influenza and red triangles for severe influenza. Distribution of ihSNV relative frequencies according to pregnancy status (P: pregnant; NP: non-pregnant) for H1N1 (C) and H3N2 (D) viruses. Severe influenza is in red. One patient treated with oseltamivir is in green.

In total, 85 non-synonymous ihSNV were detected and dispersed across the viral genome (Table S2). None of the mutations were associated with antiviral resistance. Only seven non-synonymous ihSNV (at a relative frequency between 1-10.5\%) were shared between women (Table 2). Two highly prevalent non-synonymous ihSNV (range: 9-13 patients, irrespective of pregnancy) were detected on PA (E493A) and NP (T130K) of H1N1. Five low prevalent non-synonymous ihSNV (range: 3-5 patients, irrespective of pregnancy) were detected on H1N1 HA (P182Q, T342A, E356K, HA1 numbering) and on PB1 of both H3N2 and H1N1 (K279Q, Q460K). The K279Q and Q460K substitutions on H3N2 were found exclusively in three pregnant women, while H1N1 PB1 K279Q was found in four pregnant and one non-pregnant women. However, there was no significant difference in the prevalence of these mutations or their relative frequencies according to pregnancy (Table 2). 
Table 2. Convergent non-synonymous ihSNV.

\begin{tabular}{|c|c|c|c|c|}
\hline & $\begin{array}{c}\text { Pregnant Women } \\
\quad n=36\end{array}$ & $\begin{array}{l}\text { Non-Pregnant Women } \\
\qquad n=23\end{array}$ & $\begin{array}{c}p \\
\text { Occurrence } \\
\text { of NS-ihSNV }\end{array}$ & $\begin{array}{c}p \\
\text { Relative } \\
\text { Frequencies }\end{array}$ \\
\hline \multicolumn{5}{|c|}{$\begin{array}{l}\text { H1N1, women ratio* (min-max of } \\
\text { ihSNV relative frequencies) } \\
\text { S2-PB1 }\end{array}$} \\
\hline K279Q & $4 / 6(2.3-8.6 \%)$ & $1 / 6(1.8 \%)$ & 0.24 & \\
\hline Q460K & $1 / 6(1.6 \%)$ & $0 / 6$ & 1.00 & \\
\hline \multicolumn{5}{|l|}{ S3-PA } \\
\hline E493A & 9/9 (1.3-14.3\%) & $8 / 8(1.7-10.5 \%)$ & 1.00 & 0.55 \\
\hline \multicolumn{5}{|l|}{ S4-HA** } \\
\hline P182Q & $1 / 16(4.6 \%)$ & 4/12 (1.3-3.6\%) & 0.13 & \\
\hline T342A & $2 / 13(1.2-1.4 \%)$ & 2/8 (1.2-1.7\%) & 0.53 & $>0.999$ \\
\hline E356K & $9 / 15(1.2-7.6 \%)$ & $3 / 12(1.2-6.2 \%)$ & 0.12 & $>0.999$ \\
\hline \multicolumn{5}{|l|}{ S5-NP } \\
\hline T130K & 13/17 (1.1-1.8\%) & 9/12 (1.1-3.5\%) & $>0.99$ & 0.07 \\
\hline \multicolumn{5}{|c|}{$\begin{array}{l}\text { H3N2, women ratio* (min-max of } \\
\text { ihSNV relative frequencies) } \\
\text { S2-PB1 }\end{array}$} \\
\hline K279Q & $4 / 13(1.5-3.4 \%)$ & $0 / 6$ & 0.25 & \\
\hline Q460K & $3 / 13(1.3-2.0 \%)$ & $0 / 6$ & 0.52 & \\
\hline
\end{tabular}

${ }^{*}$ Number of women with the same ihSNV on total number of women that were analysed at that position; ${ }^{* *}$ HA1 numbering; NS-ihSNV: Non-Synonymous intra-host diversity.

\section{Discussion}

In this cohort of 59 patients, we found no evidence of a specific impact of pregnancy on IAV ihD in humans. Most of the IAV infecting both pregnant and non-pregnant hospitalized women had low $\mathrm{ihD}$, with less than $1.3 \mathrm{ihSNV} / \mathrm{kb}$ per segment and relative frequencies between $1-5 \%$. None of the mutations previously found in the murine pregnancy model [3] were found, and no specific evolution of IAV during pregnancy was observed.

IAV ihD in both pregnant and non-pregnant women was very limited, which is in line with other studies showing that acute infections in immunocompetent patients are associated with less than $10 \mathrm{ihSNV}$ per sample, most at relative frequencies $1-10 \%$ [7-11]. In these patients, vaccination was not associated with increased ihD or emergence of antigenic variants $[8,10]$. In contrast, immunosuppressed patients may develop chronic IAV infection leading to increased ihD, with up to $60 \mathrm{ihSNV}$ per sample at relative frequencies $>5 \%$, including variants involved in antiviral resistance during treatment [12]. Pregnancy has been identified as a risk factor for hospitalization with influenza and consequently, vaccination of pregnant women and antiviral treatments are recommended. Our results suggest that immune modulation during pregnancy does not give rise to increased viral diversity. Therefore, infections in pregnant women are unlikely to enable the emergence of antigenic variants or resistance mutations, similarly to infections in immunocompetent patients.

This retrospective study does, however, have limitations. For instance, as only hospitalised patients were included, demographics and clinical data are not representative of the general population. In addition, only a few cases with severe influenza were included (two pregnant, five non-pregnant), limiting ihD analysis according to severity. Furthermore, only one nasopharyngeal sample/patient was sequenced at admission, while it would be interesting to compare the parallel evolution of ihD between pregnant and non-pregnant women, and between upper and lower respiratory tract. A strength of the study was that sequencing was performed in duplicate, increasing the validity of the variants identified, although very stringent criteria for ihD investigation leaded to exclusion of many samples. This is usually not done in studies of IAV quasispecies that suffer of low reproducibility for low viral loads [2].

Taken together, the results indicate that pregnancy is not associated with increased IAV ihD in humans. Although the only two pregnant women with severe influenza herein had very low IAV ihD, similar to non-pregnant women, we cannot exclude that IAV ihD may be increased in women with specific genetic or immune predisposition, but also with severe influenza. Nonetheless, the data 
presented herein suggest that the convergent mutations associated with severity in the pregnant mouse model (Q223R on HA, R211K on NS1, and R54N on NEP) [3] are not relevant in pregnant women. As these specific mutations were also observed in non-pregnant mice, although at a lower relative frequency, they might be associated with virus adaptation to mice rather than to pregnancy itself, as exemplified by Q223R. Indeed, Q223R mutation affects the receptor binding site affinity, and such mutation likely reflects IAV adaptation after inter-species infection [13,14].

Seven non-synonymous ihSNV on PA (E493A), NP (T130K) and HA (P182Q, T342A, E356K) of H1N1, and on PB1 (K279Q, Q460K) of both H3N2 and H1N1 viruses were shared by several samples. None has been reported elsewhere except for HA P182Q, detected in out- and hospitalised patients in Brazil during the 2009 pandemic [15]. None of these shared ihSNV were significantly associated with pregnancy but there was a trend towards higher occurrence of PB1 K279Q and Q460K in pregnant women. These results warrant further investigation in larger cohorts and experimental studies to assess the impact of these mutations on viral pathogenesis. Finally, while most of the ihSNV were found at different positions, we cannot exclude that some ihSNV might have similar effects on influenza virus fitness or virulence and that functional convergence might occur during influenza within-host evolution.

\section{Conclusions}

This study suggests that pregnancy does not impact IAV ihD in humans, in contrast to mice. Further studies are needed to investigate whether influenza severity in humans is associated with specific IAV within-host evolution in the lung.

Supplementary Materials: The following are available online at http://www.mdpi.com/2077-0383/8/11/1974/s1, Table S1: Sequencing performance, Table S2: Prevalence and putative functions of non-synonymous ihSNV, Figure S1: Segment coverage of H1N1 and H3N2 influenza virus genomes, Figures S2-S9: Phylogenetic tree of PB2, PB1, PA, HA, NP, NA, M1, NS1 for H1N1 (A) and H3N2 viruses (B), Figure S10. Comparison of IAV non-synonymous (NS) ihSNV between pregnant and non-pregnant women.

Author Contributions: Conceptualization, G.D. (Gregory Destras), M.P., and L.J.; methodology, G.D. (Gregory Destras), and L.J.; formal analysis, G.D. (Gregory Destras); investigation, G.D. (Gregory Destras), M.P.; resources, M.V., L.J.; writing—original draft preparation, G.D. (Gregory Destras), L.J.; writing-review and editing, G.D. (Gregory Destras), M.P., B.S., M.V., V.E., P.F., G.D. (Gil Dubernard), P.G., P.-A.B., B.L., L.J. supervision, B.L., L.J.; project administration, L.J.; funding acquisition, L.J., B.L.

Funding: This study was performed in the field of the global surveillance of influenza viruses performed by the WHO and by the "Centre National de Référence des Virus des Infections Respiratoires" supported by "Santé Publique France" (SPF) in France.

Acknowledgments: Part of the results were presented at the 21st ESCV Annual Meeting 2018 in Athens. The authors are very grateful to all clinicians, virologists, and laboratory technicians for their active participation and help in this study. We also thank Philip Robinson (DRCI, Hospices Civils de Lyon) for help in manuscript preparation.

Conflicts of Interest: B.L. is the Chair of the scientific committee of the Global Hospital Influenza Surveillance Network, the co-Chair of the Global Influenza Initiative, a member of the Foundation for Influenza. He received meeting travel grants from Sanofi-Pasteur, Seegene and Abbot Diagnostics. No personal income since 2010. L.J. has served on the French Scientific Advisory Board for SANOFI on the Influenza Vaccine in 2017, 2018, and 2019.

\section{References}

1. Domingo, E.; Sheldon, J.; Perales, C. Viral quasispecies evolution. Microbiol. Mol. Biol. Rev. 2012, 76, $159-216$. [CrossRef] [PubMed]

2. Xue, K.S.; Moncla, L.H.; Bedford, T.; Bloom, J.D. Within-Host Evolution of Human Influenza Virus. Trends Microbiol. 2018, 26, 781-793. [CrossRef] [PubMed]

3. Engels, G.; Hierweger, A.M.; Hoffmann, J.; Thieme, R.; Thiele, S.; Bertram, S.; Dreier, C.; Resa-Infante, P.; Jacobsen, H.; Thiele, K.; et al. Pregnancy-Related Immune Adaptation Promotes the Emergence of Highly Virulent H1N1 Influenza Virus Strains in Allogenically Pregnant Mice. Cell Host Microbe 2017, 21, 321-333. [CrossRef] [PubMed] 
4. Simon, B.; Pichon, M.; Valette, M.; Burfin, G.; Richard, M.; Lina, B.; Josset, L. Whole Genome Sequencing of A(H3N2) Influenza Viruses Reveals Variants Associated with Severity during the 2016-2017 Season. Viruses 2019, 11, 108. [CrossRef] [PubMed]

5. Blankenberg, D.; Von Kuster, G.; Bouvier, E.; Baker, D.; Afgan, E.; Stoler, N.; Galaxy Team; Taylor, J.; Nekrutenko, A. Dissemination of scientific software with Galaxy ToolShed. Genome Biol. 2014, 15, 403. [CrossRef] [PubMed]

6. R Core Team. R: A Language and Environment for Statistical Computing. R Foundation for Statistical Computing, Vienna, Austria. [Internet]. 2018. Available online: https://www.R-project.org/ (accessed on 12 May 2019).

7. Pichon, M.; Simon, B.; Valette, M.; Bal, A.; Picard, C.; Escuret, V.; Ottmann, M.; Gillet, Y.; Ader, F.; Lina, B.; et al. Evolution of influenza genome diversity during infection in immunocompetent patients. bioRxiv 2018, 435263.

8. Debbink, K.; McCrone, J.T.; Petrie, J.G.; Truscon, R.; Johnson, E.; Mantlo, E.K.; Monto, A.S.; Lauring, A.S. Vaccination has minimal impact on the intrahost diversity of H3N2 influenza viruses. PLoS Pathog. 2017, 13, e1006194. [CrossRef] [PubMed]

9. Sobel Leonard, A.; McClain, M.T.; Smith, G.J.D.; Wentworth, D.E.; Halpin, R.A.; Lin, X.; Ransier, A.; Stockwell, T.B.; Das, S.R.; Gilbert, A.S.; et al. Deep Sequencing of Influenza a Virus from a Human Challenge Study Reveals a Selective Bottleneck and Only Limited Intrahost Genetic Diversification. J. Virol. 2016, 90, 11247-11258. [CrossRef] [PubMed]

10. Dinis, J.M.; Florek, N.W.; Fatola, O.O.; Moncla, L.H.; Mutschler, J.P.; Charlier, O.K.; Meece, J.K.; Belongia, E.A.; Friedrich, T.C. Deep Sequencing Reveals Potential Antigenic Variants at Low Frequencies in Influenza a Virus-Infected Humans. J. Virol. 2016, 90, 3355-3365. [CrossRef] [PubMed]

11. Xue, K.S.; Bloom, J.D. Reconciling disparate estimates of viral genetic diversity during human influenza infections. Nat. Genet. 2019, 51, 1298-1301. [CrossRef] [PubMed]

12. Pichon, M.; Picard, C.; Simon, B.; Gaymard, A.; Renard, C.; Massenavette, B.; Malcus, C.; Monneret, G.; Morfin-Sherpa, F.; Valette, M.; et al. Clinical management and viral genomic diversity analysis of a child's influenza A(H1N1)pdm09 infection in the context of a severe combined immunodeficiency. Antivir. Res. 2018, 160, 1-9. [CrossRef] [PubMed]

13. Thangavel, R.R.; Bouvier, N.M. Animal models for influenza virus pathogenesis, transmission, and immunology. J. Immunol. Methods 2014, 410, 60-79. [CrossRef] [PubMed]

14. Wang, W.; Castelán-Vega, J.A.; Jiménez-Alberto, A.; Vassell, R.; Ye, Z.; Weiss, C.D. A mutation in the receptor binding site enhances infectivity of $2009 \mathrm{H} 1 \mathrm{~N} 1$ influenza hemagglutinin pseudotypes without changing antigenicity. Virology 2010, 407, 374-380. [CrossRef] [PubMed]

15. Oliveira, M.J.; Motta Fdo, C.; Siqueira, M.M.; Resende, P.C.; Born Pda, S.; Souza, T.M.; Mesquita, M.; Oliveira Mde, L.; Carney, S.; Mello, W.A.; et al. Molecular findings from influenza A(H1N1)pdm09 detected in patients from a Brazilian equatorial region during the pandemic period. Mem. Inst. Oswaldo Cruz 2014, 109, 912-917. [CrossRef] [PubMed]

(C) 2019 by the authors. Licensee MDPI, Basel, Switzerland. This article is an open access article distributed under the terms and conditions of the Creative Commons Attribution (CC BY) license (http://creativecommons.org/licenses/by/4.0/). 